\title{
Scheduling and Control Modeling of HVLV Systems Using Max-Plus Algebra
}

\author{
Imed Nasri \\ University of Savoie \\ Domaine Universitaire, BP 80439 \\ 74944 Annecy le Vieux \\ France \\ imed.nasri@univ-savoie.fr
}

\author{
Georges Habchi \\ University of Savoie \\ Domaine Universitaire, BP 80439 \\ 74944 Annecy le Vieux \\ France \\ georges.habchi@univ-savoie.fr
}

\author{
Reda Boukezzoula \\ University of Savoie \\ Domaine Universitaire, BP 80439 \\ 74944 Annecy le Vieux \\ France \\ reda.boukezzoula@univ-savoie.fr
}

\begin{abstract}
The High-Variety, Low-Volume (HVLV) scheduling problem is one of the most arduous and combinatorial optimization problems. This paper presents an analytical scheduling model using a tropical algebra called $(\max ,+)$ algebra. The aim is to find an allocation for each operation and to define the sequence of operations on each machine, so that the resulting schedule has a minimal completion time and the due dates of the different jobs (products) are met such that a Just-In-Time (JIT) production will be satisfied. To generate feasible schedules, decision variables are introduced in the model. The algebraic model developed in this 'work describes the discontinuous operations aspect of HVLV systems as Discrete Event Dynamic Systems (DEDS). It is non-linear in the sense of $(\max ,+)$ algebra. The focus of this research concerns the development of a static scheduling approach for deterministic and not-decision-free HVLV manufacturing systems. Firstly, using (max, +) algebra, a direct generation of event-timing equations for deterministic and not-decision free HVLV systems is obtained. Then, a non-linear optimization problem in (max, + ) algebra is solved. Finally, the validity of the proposed approach is illustrated by simulation examples.
\end{abstract}

optimization

HVLV manufacturing systems, (max, +) non-linear model, scheduling and control modeling, non-linear

\section{INTRODUCTION}

High-Variety, Low-Volume (HVLV) manufacturing systems are a class of dynamic systems where the behavior is similar to Discrete Event Dynamic Systems (DEDS). They are characterized by a wide variety of products using shared machines, a weak and personalized demand, relatively long processing times and frequent change over and set-up times. Consequently, a continuous approximation of the production flow by continuous flow systems (Habchi and Berchet, 2003) (Tamani, Boukezzoula and Habchi, 2009) (Tamani, Boukezzoula and Habchi, 2011) is not appropriate for HVLV systems. In this framework, it seems very interesting to handle this kind of systems as Job-Shop systems (Huang and Irani, 2003) due to the wide variety of processed products.

Production systems can be modeled from two different points of view, qualitative or quantitative. Qualitative models capture logical aspects of a system such us deadlocks, conflicts, etc. Automata and finite state machines (Hopcroft, Motwani and Ullman, 1979) and Petri Nets (PN) called also untimed models (Kasturia, Dicesare and Desrochers, 1988) fall into this category. A PN is a graphical technique that allows the modeling of systems with parallel and synchronous processes. The model may then be used for the analysis, the representation of the dynamic evolution of the system and for the controller synthesis. Quantitative models, highlight the quantitative system performance in terms of the throughput, number of parts in the system (work-inprocess), due dates of products, etc. Discrete event simulation and (max, +) algebra (Baccelli, Cohen and Quadrat, 1992) are examples of quantitative models.

The work presented in this paper is a part of quantitative modeling. The main goal is to propose an analytical (max, +) model and develop a procedure able to generate the event-timing equations. These equations are elaborated from a not-decision-free Job-Shop system configuration. A system where the concurrent operations processed 
on the same machine need a decision to solve the conflicts.

Decision-free DEDS with only synchronization has been modeled in dioid algebra by (Cohen, Dubois, Quadrat and Viot, 1985) (Engell, 1989) (Nasri, Boukezzoula and Habchi, 2011). The different proposed models are linear in dioid algebra. In this study, control variables are introduced to the model to make sequencing decisions. (Baccelli, Cohen and Quadrat, 1992) noted that non-linear equations are needed in dioid algebra to model the scheduling problem.

The dioid algebraic model developed in this research must generate all feasible schedules by choosing different values for decision variables. This model is non-linear in the sense of $(\max ,+)$ algebra.

Job-Shop scheduling problems belong to the class of combinatorial problems and are one of the most difficult problems to solve. They are proved to be NP-hard (Tamaki and Sannomiya, 1998) (Beatrice and Mario, 2004) (Wang and Tang, 2011). A nonlinear optimization in (max, +) algebra using global programming solver is considered in this work.

The remainder of this paper is organized as follows: Section 2 describes a state-space HVLV systems scheduling modeling. Section 3 shows an illustrative example of the scheduling modeling of a $(6 \times 6)$ JobShop using $(\max ,+)$ algebra. Next, a nonlinear optimization technique in $(\max ,+)$ algebra is applied to minimize the makespan and the total tardiness subject to JIT production. Concluding remarks and future research directions are presented in section 5.

\section{HVLV SYSTEMS SCHEDULING MODELING}

The focus of this section concerns the development of an approach to generate event-timing equations directly from the machine interconnections for a deterministic and not-decision free Job-Shop HVLV system.

As mentioned above, the DEDS models developed so far can be classified into two major categories: untimed and timed models (Table 1). Untimed models emphasize state or event sequences of a DEDS and ignore the holding times of a system in every state or event. Such models like PN are usually employed in order to answer questions of a logical or qualitative nature. Timed models, on the other hand, are used to answer questions which relate to performance (mean holding times, meeting due dates of products, etc). Timed petri nets are an example of these models (Baccelli, Cohen and Quadrat, 1992) where it is necessary to obtain a digraph before the timing equations can be obtained. However, these approaches cannot model a real scheduling for not-decisionfree manufacturing systems. Moreover, it can be difficult since their complexities increase rapidly as the number of machines and/or the number of operations processed by machines in a production system is increased.

Table 1: Classification of DEDS models

\begin{tabular}{|l|l|l|}
\hline & Timed & Untimed \\
\hline Logical & $\begin{array}{l}\text { Timed Petri } \\
\text { Nets }\end{array}$ & Petri Nets \\
\hline Algebraic & Dioid & \\
& Algebra & \\
\hline Performance & Queuing & \\
& Networks, & \\
& Markov & \\
& Chains, & \\
& Simulation & \\
\hline
\end{tabular}

Algebraic models can be a good solution to overcome the above problem. Also, they provide many attractive features: they aim to capture the description of the trajectories of manufacturing systems in terms of a set of operations on functions of state and/or events. Baccelli et al (Baccelli, Cohen and Quadrat, 1992) and Engell (Engell, 1989) have been demonstrated that the representation of decision-free manufacturing systems using dioid algebra is similar to the one employed in Continuous Variable Dynamic Systems (CVDS) by differential equations and algebraic operations on functions of state and inputs (Tamani, Boukezzoula and Habchi, 2009). The models obtained are linear in the sense of $(\max ,+)$ algebra. Then, this representation of decision-free systems allows the formulation and the application of control concepts such as, tracking problems, predictive control (Schutter and Boom, 2003) (Schutter and Boom, 2004), stability, controllability, and observability in a similar manner to that of CVDS. However, the linear $(\max ,+)$ models proposed in the literature cannot represent a feasible scheduling for not-decisionfree manufacturing systems. Consequently, the nonlinearity in $(\max ,+)$ algebra is needed to model the scheduling problem.

\subsection{Approach Principle}

The approach presented in this paper is a direct systematic procedure applicable to a wide class of manufacturing systems, especially HVLV systems. The technique to solve the scheduling problem of HVLV systems considered the problem as a mathematical programming formulation with the using of decision variables. These control variables introduce a non-linearity in the proposed model due 
to their multiplication by the state variables (starting times of operations). They are used to solve the conflicts between concurrent operations processed on the same machine (Table 2).

Table 2: Different forms of (max, + ) models

\begin{tabular}{|l|l|l|}
\hline $\begin{array}{l}\text { Manufacturing } \\
\text { systems }\end{array}$ & $\begin{array}{l}\text { Linear (max, } \\
+ \text { model }\end{array}$ & $\begin{array}{l}\text { Non-linear } \\
\text { (max, } \\
\text { model }\end{array}$ \\
\hline $\begin{array}{l}\text { Decision- } \\
\text { free systems }\end{array}$ & $\begin{array}{l}\text { Application } \\
\text { of } \\
\text { conventional } \\
\text { control } \\
\text { techniques } \\
\text { (reference } \\
\text { tracking } \\
\text { problems, } \\
\text { internal } \\
\text { model } \\
\text { control, } \\
\text { predictive } \\
\text { control, etc.) }\end{array}$ & \\
\hline $\begin{array}{l}\text { Not- } \\
\text { decision- } \\
\text { free systems }\end{array}$ & & $\begin{array}{l}\text { Scheduling } \\
\text { problems } \\
\text { solving }\end{array}$ \\
\hline
\end{tabular}

Max-plus algebra is applied here as the modelling tool in order to represent the scheduling problem of HVLV systems where relationships between the starting times of the operations require the maximum and addition operators. The control variables used in the proposed model in the case of minimization of the makespan are the decision variables (Subsection 2.2) in order to generate feasible schedules on machines. In the case of minimization of the total tardiness, the control variables used in the proposed model are the decision variables to generate sequencing and the instants at them raw materials are fed as late as possible to the system (JIT production) to meet due dates of products. As a consequence, the internal buffer levels are kept as low as possible.

However, for the purpose of the mathematical development we consider the following assumptions:

- A Job-Shop HVLV system is considered.

- The system is deterministic and not-decisionfree.

- The number of jobs to be processed over the planning horizon is defined in advance.

- Each machine can perform one operation at a time.

- Routing decisions are already made and each operation is assigned to a particular machine.
- An operation can start only when all other events have to be performed before that particular operation have finished and the necessary resources are available. A machine can start processing on a new job as soon as that machine completes processing its current running job and there is a job ready to be processed in the queue.

A dioid is considered as a set $D$ with two operators, $\oplus$ and $\otimes$. The operation $\oplus$ called addition, produces in $\mathrm{D}$ a structure of a commutative monoid and has a neutral element $\epsilon$ called zero. The other operation, $\otimes$, called multiplication, produces in $D$ a structure of a monoid and has a neutral element $e$, called unity. Max-plus is a dioid, which consists of the real numbers $R$ extended to include $-\infty$. Max-plus algebra is used in development of algebraic models of DEDS (Baccelli, Cohen and Quadrat, 1992). For all $a, b \in R \cup-\infty$ the max-plus operators are defined according to the following equations:

$$
\begin{gathered}
a \oplus b=\max (a, b) \\
a \otimes b=a+b
\end{gathered}
$$

\subsection{Max-Plus Scheduling Model for HVLV Systems}

The goal here is to establish a $(\max ,+)$ algebraic model for the static scheduling problem for the HVLV systems. The knowledge of the individual operations for each job, the machines on which each operation should be executed, and the predecessors and external starting conditions of each operation (i.e., the process plan for each job, the instants at them raw materials are fed to the system and the starting date of a new scheduling in a new planning horizon) provide all the information needed to establish the model.

The model contains three parts: the first part considers initial conditions (exogenous conditions of the beginning of the scheduling), the second part describes precedence requirements of the operations and the third part incorporates decision variables which generate all feasible sequencing on the same machines in our system (Figure 1).

Within this context, one can then proceed to develop a (max, +) algebraic state space model for the static scheduling problem for HVLV systems. Let M denote the set of all machines available in the system, $\mathrm{N}$ the set of all jobs (products) introduced to the system and $P$ the set of all the operations on the jobs over the planning horizon. Let $x_{i j k}$ be the start time of the operation $j$ for the job (product) $i$ on machine $k$, then the completion time of operation $j$ for the job $i$ on 


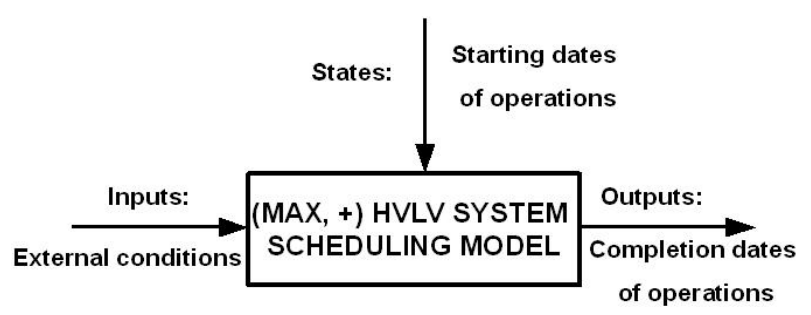

Figure 1: HVLV systems modeling principle.

machine $k$ is equal to its processing time $p_{i j k}$ plus its start time $x_{i j k}$, i.e., $x_{i j k}+p_{i j k}$. Two situations may arise depending on whether operation $j$ is the first operation on the job (has no predecessors) or that operation $j$ has some given predecessors.

If operation $j \in P$ is the first (i.e., unprecedented) operation on the job, then its processing start time $x_{i j k}$ is determined by the maximum of either:

- The starting date $t$ of the new scheduling over the new planning horizon,

- The date $u_{i}$ at which the raw material of its corresponding product $i$ is fed to the system,

- The completion of other operations $\left(j^{\prime} \neq j\right.$, and $j^{\prime} \in P$ ), from other products $i^{\prime}$ that require processing on machine $k$. This is determined by the decision variables $V_{i j k, i^{\prime} j^{\prime} k}$ that determine which operation must be processed earlier on machine $k$.

An expression for this situation may be written mathematically as follow:

$$
x_{i j k}=\max \left(t ; u_{i} ; p_{i^{\prime} j^{\prime} k}+x_{i^{\prime} j^{\prime} k}+V_{i j k, i^{\prime} j^{\prime} k}\right)
$$

Using dioid notation, the above expression may be written as:

$$
x_{i j k}=t \oplus u_{i} \oplus p_{i^{\prime} j^{\prime} k} \otimes x_{i^{\prime} j^{\prime} k} \otimes V_{i j k, i^{\prime} j^{\prime} k}
$$

In the second situation, if operation $j \in P$ is not the starting operation (i.e., has predecessors) on the job, then its processing start time $x_{i j k}$ is determined by the maximum of either:

- The completion time of its direct predecessor, say $n \in P$, being processed on its correspondent machine, say $m \in M$,

- The completion of other operations $\left(j^{\prime} \neq j\right.$, and $j^{\prime} \in P$ ), from other products $i^{\prime}$ that require processing on machine $k$. This is determined by the decision variables $V_{i j k, i^{\prime} j^{\prime} k}$ that determine which operation must be processed earlier on machine $k$.

An expression for this situation may be written mathematically as follow:

$$
x_{i j k}=\max \left(p_{i n m}+x_{i n m} ; p_{i^{\prime} j^{\prime} k}+x_{i^{\prime} j^{\prime} k}+V_{i j k, i^{\prime} j^{\prime} k}\right)
$$

Using dioid notation, the above expression may be written as:

$$
x_{i j k}=p_{i n m} \otimes x_{i n m} \oplus p_{i^{\prime} j^{\prime} k} \otimes x_{i^{\prime} j^{\prime} k} \otimes V_{i j k, i^{\prime} j^{\prime} k}
$$

The above model is non-linear in $(\max ,+)$ algebra due to the multiplication in dioid algebra between the state and control variables. The incorporation of the decision variables in the model stems from the fact that the sequencing of operations from different products on the same machine requires a decision on the order in which the operations are processed on the machine such the conflicts are resolved and precedence constraints are not violated.

To get feasible schedules, constraints are needed to bound decision variables and these constraints must satisfy the following conditions:

$$
V_{i j k, i^{\prime} j^{\prime} k}+V_{i^{\prime} j^{\prime} k, i j k}=B
$$

$$
\max \left(V_{i j k, i^{\prime} j^{\prime} k} ; V_{i^{\prime} j^{\prime} k, i j k}\right)=0
$$

where $B$ is chosen small enough and $V_{i j k, i^{\prime} j^{\prime} k}$ and $V_{i^{\prime} j^{\prime} k, i j k}$ are less than or equal to zero.

Or, using $(\max ,+)$ algebra notation,

$$
V_{i j k, i^{\prime} j^{\prime} k} \otimes V_{i^{\prime} j^{\prime} k, i j k}=B
$$

$$
V_{i j k, i^{\prime} j^{\prime} k} \oplus V_{i^{\prime} j^{\prime} k, i j k}=0
$$

The two above constraints mean that: if $V_{i j k, i^{\prime} j^{\prime} k}=$ $B$, then $V_{i^{\prime} j^{\prime} k, i j k}=0$ and if $V_{i j k, i^{\prime} j^{\prime} k}=0$, then $V_{i^{\prime} j^{\prime} k, i j k}=B$. 
Within this context, if $V_{i j k, i^{\prime} j^{\prime} k}=B$, and referring to the equations 3 or 5 in the above model, then operation $j \in P$ is processed before operation $j^{\prime} \in P$ on machine $k \in M$. This stems from the fact that the term $p_{i^{\prime} j^{\prime} k}+x_{i^{\prime} j^{\prime} k}+V_{i j k, i^{\prime} j^{\prime} k}$ in 3 and 5 will be eliminated. If $V_{i j k, i^{\prime} j^{\prime} k}=0$, then then operation $j \in P$ is processed after operation $j^{\prime} \in P$ on machine $k \in M$.

\section{ILLUSTRATIVE EXAMPLE}
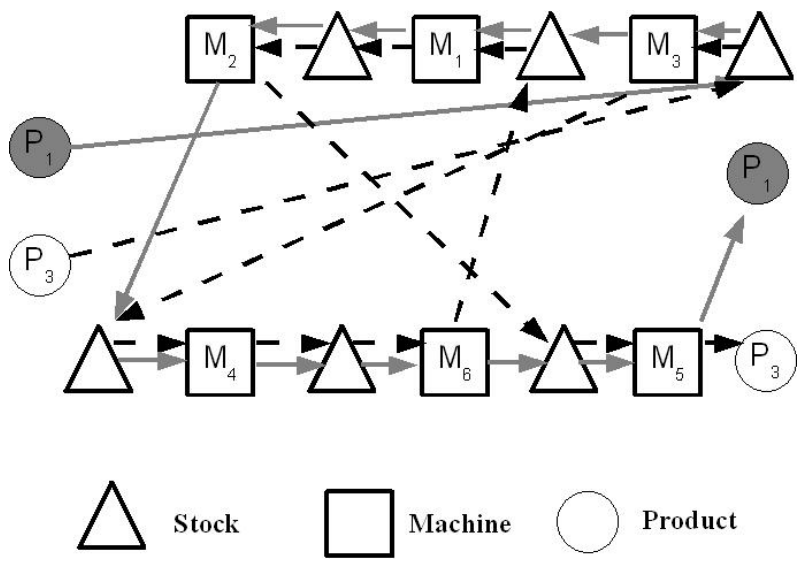

Figure 2: Job-shop HVLV system.

The application of the $(\max ,+)$ model proposed in the above section is explored below with an example of (6x6) Job-Shop system (6 products and 6 machines) (Figure 2).

This example is taken from a real factory environment (Wang and Tang, 2011). It describes very well a HVLV system due to the wide variety of products (six kinds of products) and the processing times which are relatively long. Data about routing and processing times for the 6 products are presented in Table 3.

\section{Table 3: Production data}

\begin{tabular}{|c|c|}
\hline job & Sequence (machine number, processing tim \\
\hline J1 & $1(3,1) 2(1,3) \quad 3(2,6) \quad 4(4,7) 5(6,3) \quad 6(5,6)$ \\
\hline J2 & $1(2,8) \quad 2(3,5) \quad 3(5,10) \quad 4(6,10) \quad 5(1,10) \quad 6(4,4)$ \\
\hline J3 & $1(3,5) 2(4,4) 3(6,8) 4(1,9) 5(2,1) 6(5,7)$ \\
\hline $\mathrm{J} 4$ & $1(2,5) 2(1,5) \quad 3(3,5) \quad 4(4,3) 5(5,8) 6(6,6)$ \\
\hline J5 & $1(3,9) \quad 2(2,3) \quad 3(5,5) \quad 4(6,4) \quad 5(1,3) \quad 6(4,1)$ \\
\hline J6 & $1(2,3) \quad 2(4,3) \quad 3(6,9) \quad 4(1,10) \quad 5(5,4) \quad 6(3,1)$ \\
\hline
\end{tabular}

In this example, the operations of six jobs are scheduled on six machines (Table 3 ) and we have 36 state variables $x_{i j k}(i=1: 6, j=1: 36$ and $k=1: 6$ ). For the sake of simplicity and without loss of generality, to illustrate the proposed model, we consider only the first operation $O_{113}$ of product $J 1$ and the third operation $O_{235}$ of product $J 2$. The operation $O_{113}$ is processed on machine $k=3$. It has no predecessor. The operation $O_{235}$ is manufactured on machine $k=5$. It has two predecessors: the first one is $O_{212}$ which is processed on machine $k=2$ and the second one is $\mathrm{O}_{223}$ which is processed on machine $k=3$. Then, the dynamic equations of the starting times of operations $O_{113}$ and $O_{235}$ can be described by the following $(\max ,+)$ equations referring to the model proposed in section 2:

$$
\begin{aligned}
& x_{113}=\max \left(t ; u_{1} ; p_{223}+x_{223}+V_{113,223} ; p_{313}\right. \\
& +x_{313}+V_{113,313} ; p_{433}+x_{433} \\
& +V_{113,433} ; p_{513}+x_{513}+V_{113,513} \\
& \left.p_{663}+x_{663}+V_{113,663}\right) \\
& x_{235}=\max \left(p_{223}+x_{223} ; p_{165}+x_{165}\right. \\
& +V_{235,165} ; p_{365}+x_{365}+V_{235,365} ; \\
& p_{455}+x_{455}+V_{235,455} ; p_{535}+x_{535} \\
& \left.+V_{235,535} ; p_{655}+x_{655}+V_{235,655}\right)
\end{aligned}
$$

or, using dioid notation the above equations can be written as:

$$
\begin{aligned}
x_{113}= & t \oplus u_{1} \oplus p_{223} \otimes x_{223} \otimes V_{113,223} \oplus p_{313} \\
& \otimes x_{313} \otimes V_{113,313} \oplus p_{433} \otimes x_{433} \\
& \otimes V_{113,433} \oplus p_{513} \otimes x_{513} \otimes V_{113,513} \oplus \\
& p_{663} \otimes x_{663} \otimes V_{113,663} \\
x_{235}= & p_{223} \otimes x_{223} \oplus p_{165} \otimes x_{165} \\
& \otimes V_{235,165} \oplus p_{365} \otimes x_{365} \otimes V_{235,365} \oplus \\
& p_{455} \otimes x_{455} \otimes V_{235,455} \oplus p_{535} \otimes x_{535} \\
& \otimes V_{235,535} \oplus p_{655} \otimes x_{655} \otimes V_{235,655}
\end{aligned}
$$

Note that all the dynamic equations of the remainder operations in the example can be obtained in the same way as the two above equations.

The dynamic of the system equation can be grouped in $(\max ,+)$ matrix form:

$$
X=T \oplus U \oplus C \otimes X
$$

where: $X$ is a $(36 \times 1)(\max ,+)$ state vector which collects the starting times of operations, $T$ is a $(36 \times 1)(\max ,+)$ vector which is composed of the 
beginning dates of the scheduling over the new planning horizon, $U$ is a (36x1) vector which contains the different dates at which the raw material of each product is fed to the system and $C$ is a $(36 \times 36)$ appropriate $(\max ,+)$ matrix describing the relationships among different state variables of the system. It contains the different decision variables.

\section{APPLICATION OF THE (MAX, +) MODEL FOR THE SCHEDULING OF HVLV SYSTEMS: NONLINEAR OPTIMIZATION}

In this section, the dioid algebraic model is used to develop the static scheduling of the system. Then, a non-linear optimization problem in $(\max ,+)$ algebra is applied to minimize the makespan and the total tardiness subject to JIT production.

\subsection{Non-linear Optimization for the Makespan Minimization}

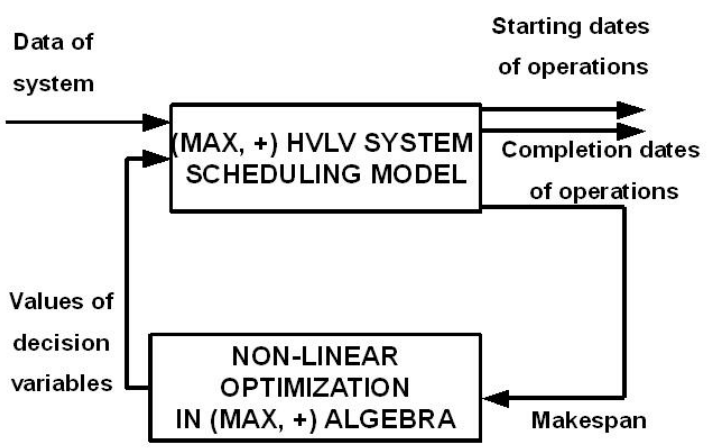

Figure 3: Full scheme of makespan optimization.

The objective of this section is to minimize the makespan using a non-linear optimization in (max, +) algebra (Figure 3)

Let define the makespan criterion of the HVLV system as follow:

$$
C_{\max }=\max \left(C_{i}\right)=\max \left(x_{i w k}+p_{i w k}\right)
$$

where $C_{i}$ is the completion time of product $i$ and $w$ is the last operation of product $i$.

or, using the $(\max ,+)$ notation :

$$
C_{\text {max }}=\bigoplus_{i=1}^{n} C_{i}=\bigoplus_{i=1}^{n}\left(x_{i w k} \otimes p_{i w k}\right)
$$

where $x_{i w k}$ is the starting time of the last operation $w$ of product $i$ on machine $k$ and $p_{i w k}$ is its corresponding processing time. Then, the nonlinear optimization scheduling problem into ( $\max ,+$ ) algebra is defined as:

$$
C_{\max }^{*}=\min C_{\max }=\min \left(\max \left(x_{i w k}+p_{i w k}\right)\right)
$$

Subject to:

$$
V_{i j k, i^{\prime} j^{\prime} k}+V_{i^{\prime} j^{\prime} k, i j k}=B
$$

$$
\max \left(V_{i j k, i^{\prime} j^{\prime} k} ; V_{i^{\prime} j^{\prime} k, i j k}\right)=0
$$

$$
\begin{gathered}
V_{i j k, i^{\prime} j^{\prime} k} \leqslant 0 \\
V_{i^{\prime} j^{\prime} k, i j k} \leqslant 0 \\
B \lll 0
\end{gathered}
$$

$$
x_{i j k}=\max \left(p_{i n m}+x_{i n m} ; p_{i^{\prime} j^{\prime} k}+x_{i^{\prime} j^{\prime} k}+V_{i j k, i^{\prime} j^{\prime} k}\right)
$$

where $j=1: w$ and $w$ is the number of operations in each product $i$.

Applying the above non-linear optimization problem to the example of (6x6) Job-Shop HVLV system shown in section 3 with $t=u_{i}=0$ for $i=$ 1 : 6 . Then, the obtained optimal value $C_{\max }^{*}=$ $\max \left(x_{165}+p_{165} ; x_{264}+p_{264} ; x_{365}+p_{365} ; x_{466}+\right.$ $\left.p_{466} ; x_{564}+p_{564} ; x_{663}+p_{663}\right)=55$ time units. The corresponding schedules on the different machines based on the proposed $(\max ,+)$ model are showed in Figure 4 that shows the order of each job $J i$ on each machine $k$.

The completion times $C_{i}$ of the different products $i=1: 6$ are presented in Table 4 . 


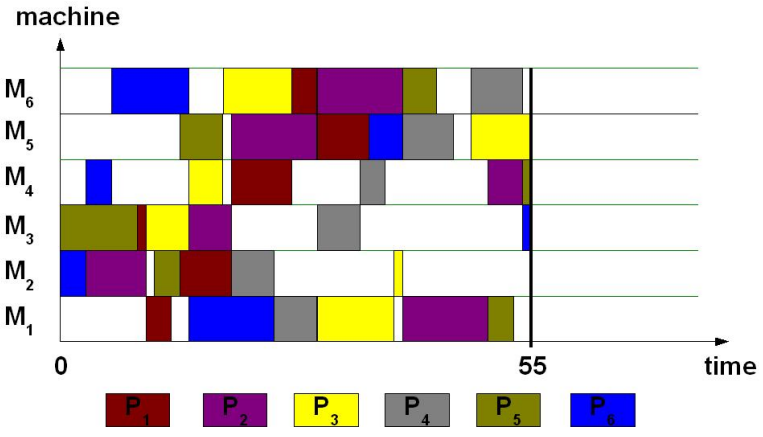

Figure 4: Operations scheduling on the machines.

Table 4: Completion times of jobs

\begin{tabular}{|c|c|c|c|c|c|c|}
\hline Jobs & $\mathrm{J} 1$ & $\mathrm{~J} 2$ & $\mathrm{~J} 3$ & $\mathrm{~J} 4$ & $\mathrm{~J} 5$ & $\mathrm{~J} 6$ \\
\hline$C_{i}$ & 36 & 54 & 55 & 54 & 55 & 55 \\
\hline
\end{tabular}

\subsection{Results Discussion}

As mentioned in Subsection 4.1, the proposed model associated to a non-linear optimization algorithm in $(\max ,+)$ algebra leads to an optimal value of the makespan $C_{\max }^{*}=55$ time units. A little comparison between our scheduling technique and the two methods proposed in the literature (Wang and Tang, 2011) to minimize the makespan, shows the same values of the optimal makespan (55 time units) (Figure 5).

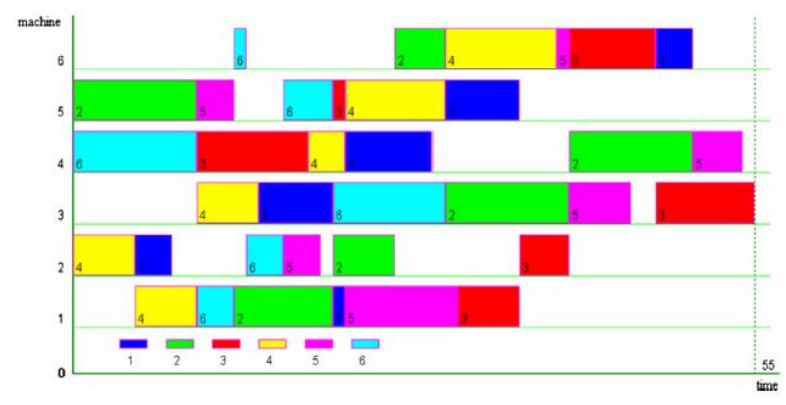

Figure 5: Makespan minimization (Wang and Tang, 2011).

In (Wang and Tang, 2011), the authors have been used the traditional Genetic Algorithm (GA) and an Improved Adaptive Genetic Algorithm (IAGA) for solving the minimum makespan problem of the jobshop scheduling problem presented in Table 3 . In the proposed non-linear $(\max ,+)$ scheduling model, we have not parameters to tun which is the case in (Wang and Tang, 2011) (initial population chose, crossover probability and mutation probability tuning). With a such model, we must only choose the suitable mathematical programming formulation of the scheduling problem and the appropriate decision variables to generate different feasible schedules.

\subsection{Non-linear Optimization for the Total Tardiness Minimization Subject to JIT Production}

The objective of this section is to minimize the total tardiness criterion for a non-linear optimization using the $(\max ,+)$ algebra and subject to JIT production (Figure 6)

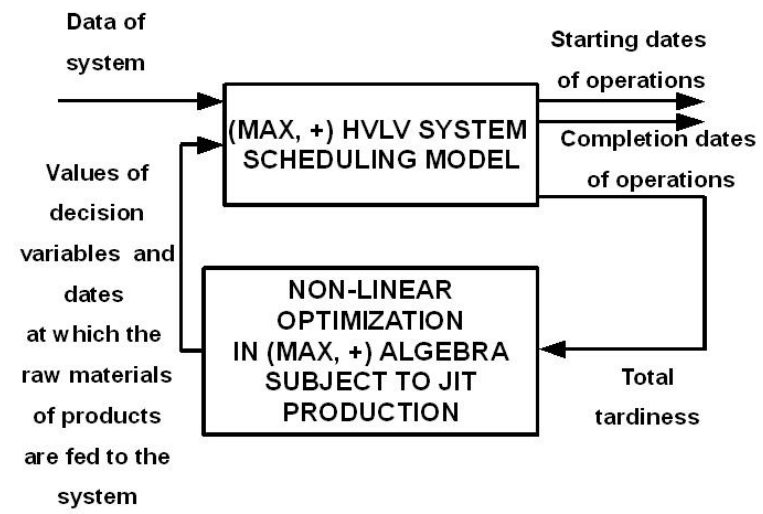

Figure 6: Full scheme of total tardiness optimization.

As far as we know, there are few researches about scheduling problems in the literature that deal with the total tardiness minimization. Moreover, all these researches don't handle the JIT production criterion in the scheduling problems. In this section, the total tardiness is minimized, so that the JIT production is satisfied. Then, let define the following criterion:

$$
T_{d}+T_{e}
$$

or, using the $(\max ,+)$ notation :

$$
T_{d} \otimes T_{e}
$$

where :

$$
T_{d}=\sum_{i=1}^{n} T_{i}
$$

and

$$
T_{e}=-\sum_{i=1}^{n} u_{i}
$$

or, using the $(\max ,+)$ notation : 


$$
T_{d}=\bigotimes_{i=1}^{n} T_{i}
$$

and

$$
T_{e}=-\bigotimes_{i=1}^{n} u_{i}
$$

Note that $n$ is the number of products and $T_{i}=$ $\max \left(x_{i w k}+p_{i w k}-D_{i} ; 0\right)$, for $i=1: n$, and $k=1: m$ ( $k$ is the number of machines).

Where $w$ is the last operation of product $i$ and $x_{i w k}$ is the starting time of the last operation $w$ of product $i$ on machine $k$ and $p_{i w k}$ is its corresponding processing time. $D_{i}$ is the due date of the product $i$.

- $T_{d}$ reflects the due date tracking error,

- $T_{e}$ reflects the control effort (JIT criterion). The minimization of $T_{e}$ would lead to maximization of the dates $u_{i}$ at which the raw material of each product is fed to the system as late as possible. Consequently, the starting time of the first operation $x_{i 1 k}$ of each product $i$ will be equal to $u_{i}$.

Then, the non-linear optimization scheduling problem into $(\max ,+)$ algebra is defined as:

$$
T^{*}=\min T=\min \left(T_{d}+T_{e}\right)
$$

Subject to:

$$
\begin{gathered}
V_{i j k, i^{\prime} j^{\prime} k}+V_{i^{\prime} j^{\prime} k, i j k}=B \\
\max \left(V_{i j k, i^{\prime} j^{\prime} k} ; V_{i^{\prime} j^{\prime} k, i j k}\right)=0 \\
V_{i j k, i^{\prime} j^{\prime} k} \leqslant 0 \\
V_{i^{\prime} j^{\prime} k, i j k} \leqslant 0 \\
B 0
\end{gathered}
$$

$$
x_{i j k}=\max \left(t ; u_{i} ; p_{i^{\prime} j^{\prime} k}+x_{i^{\prime} j^{\prime} k}+V_{i j k, i^{\prime} j^{\prime} k}\right)
$$

$$
x_{i j k}=\max \left(p_{i n m}+x_{i n m} ; p_{i^{\prime} j^{\prime} k}+x_{i^{\prime} j^{\prime} k}+V_{i j k, i^{\prime} j^{\prime} k}\right)
$$

$$
C_{i}=x_{i j k}+p_{i j k} \leqslant D_{i}
$$

where $C_{i}$ is the completion time of product $i$.

The above non-linear optimization problem is applied to the example of (6x6) Job-Shop HVLV system shown in section 3 with $t=0$ and for the different chosen due dates $D_{i}$ (Table 5).Then, the obtained optimal value $T^{*}=-80$ time units. This optimal criterion corresponds to the following completion times of products (Table 5):

Table 5: Due dates and completion times of jobs

\begin{tabular}{|c|c|c|c|c|c|c|}
\hline Jobs & $\mathrm{J} 1$ & $\mathrm{~J} 2$ & $\mathrm{~J} 3$ & $\mathrm{~J} 4$ & $\mathrm{~J} 5$ & $\mathrm{~J} 6$ \\
\hline$D_{i}$ & 60 & 77 & 55 & 36 & 45 & 55 \\
\hline$C_{i}$ & 60 & 77 & 40 & 36 & 43 & 55 \\
\hline
\end{tabular}

The corresponding scheduling on the different machines based on the proposed $(\max ,+)$ model is as follow (Figure 7).

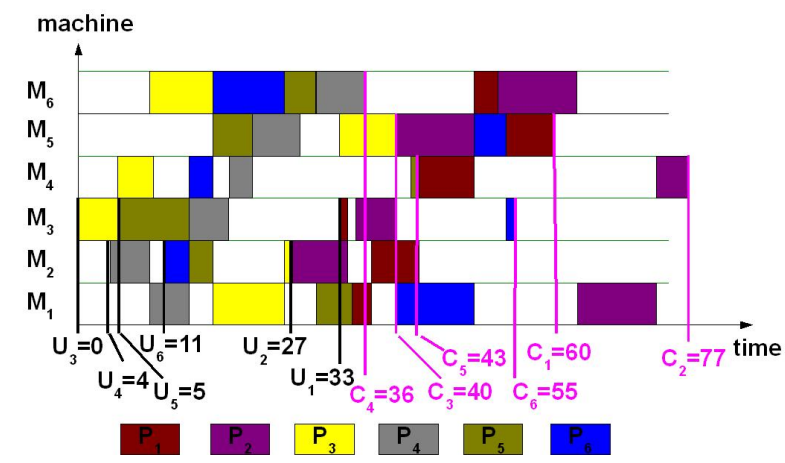

Figure 7: Operations scheduling on the machines.

Table 6: Controlled dates of the first operation of the products

\begin{tabular}{|c|c|c|c|c|c|c|}
\hline Jobs & J1 & J2 & J3 & J4 & J5 & J6 \\
\hline$u_{i}$ & 33 & 27 & 0 & 4 & 5 & 11 \\
\hline$x_{i 1 k}$ & 33 & 27 & 0 & 4 & 5 & 11 \\
\hline
\end{tabular}

\subsection{Results Discussion}

Table 5 shows that the due dates $D_{i}(i=1: 6)$ of the different products are met. Then for all $i=1: 6, C_{i} \leqslant$ $D_{i}$. Moreover, Table 6 shows that the JIT production 
criterion is satisfied. Effectively, the starting times of each first operation of the product $i, x_{i 1 k}$, is equal to the date at which the raw material of each product $i$, $u_{i}$, is fed to the system. Then, the proposed criterion $T e$ allows the maximization of the input time instants $u_{i}$, so that the raw material of products is fed to the system as late as possible. As a consequence, the internal buffer levels are kept as low as possible.

\section{CONCLUSIONS}

This work was directed towards extending $(\max ,+)$ algebraic applications for scheduling, optimization, and control of HVLV systems. Linear (max, +) models cannot represent a scheduling problem for a not-decision-free systems. The non-linearity into dioid algebra is needed for HVLV systems scheduling. The proposed model incorporates decision (control) variables as conflicts resolvers between concurrent operations on the same machine. Non-linear optimization problem is then solved into $(\max ,+)$ algebra to minimize two criteria. The total tardiness criterion is extended to solve a JIT production problem. The simulation results show that the proposed model can be a good tool for the control and optimization of HVLV systems.

In real-world applications for HVLV systems, various uncertainty aspects of the system will perturb its behavior (machines breakdowns, set-up times, etc). In this context, next research work will be done to improve the $(\max ,+)$ model such that it can deal with a dynamic HVLV systems scheduling.

\section{REFERENCES}

Baccelli, F., Cohen, G. and Quadrat, J.P. (1992) Synchronisation and linearity. Wiely New York.

Beatrice, M. and Mario, V. (2004) Local search genetic algorithms for job-shop scheduling problem. Applied Intelligence, 21(1): 99-110.

Cohen, G., Dubois, D. and Quadrat, J.P. (1985) A linear-system-theoretic view of discrete-event processes and its use for performance evaluation in manufacturing. IEEE transactions on Automatic Control, AC-30: 210-110.

Engell, S. (1989) A decentralized on-line scheduling strategy for FMS. In 6th IFAC/IFIP/IFORS/IMACS Symposium on information Control Problems in Manufacturing technology, Madrid, Spain, 26-29 September 1989, page 437.

Habchi, G. and Berchet, C. (2003) A model for manufacturing systems simulation with a control dimension. Simulation Modelling Practice and Theory, 11(1): 21-44.
Hopcroft, J., Motwani, R. and Ullman, J. (1979) Introduction to automata theory, languages, and computation (2nd edn). Addison-Wesley.

Huang, H. and Irani, S. (2003) An enhanced systematic layout planning process for high-variety low-volume (HVLV) manufacturing facilities. In 17th international conference on Production research, Virginia, USA, 3-7 August 2003.

Kasturia, E., Dicesare, F. and Desrochers, A. (1988) real time control of multilevel manufacturing systems using colored petri nets. In IEEE international Conference on Robotics and Automation.

Nasri, I., Boukezzoula, R. and Habchi, G. (2011) A propos de la modélisation et le pilotage des systèmes manufacturiers de type HVLV. In 12ème Congrès Annuel de la Société Francaise de Recherche Opérationnelle et d'aide à la Décision, Saint-Etienne, France, 2-4 March 2011, N383, page II-665.

Schutter, B.D. and Van den Boom, T. (2003) MPC for discrete event systems with soft and hard synchronization constraints. International Journal of Control, 76(1): 82-94.

Schutter, B.D. and Van den Boom, T. (2004) MPC for continuous piecewise-affine systems. Systems Control Letters, 52(3-4): 179-192.

Tamaki, H. and Sannomiya, N. (1998) Genetic algorithms and neural networks. Corona Publishing.

Tamani, K., Boukezzoula, R. and Habchi, G. (2009) Intelligent distributed and supervised flow control methodology for production systems. Engineering Applications of Artificial Intelligence, 22(7): 11041116.

Tamani, K., Boukezzoula, R. and Habchi, G. (2011) Multi-objective supervisory flow control based on fuzzy interval arithmetic: Application for scheduling of manufacturing systems. Simulation modelling Practise and Theory, 19(5): 1371-1383.

Wang, I. and Tang, D. (2011) an improved adaptive genetic algorithm based on hormone modulation mechanism for job-shop scheduling problem. Expert Systems with Application, 38(6): 7243-7250. 